\title{
Infrared steam laser cleaning
}

\author{
P. Frank • F. Lang $\cdot$ M. Mosbacher $\cdot$ J. Boneberg • \\ P. Leiderer
}

\begin{abstract}
Steam Laser Cleaning with a pulsed infrared laser source is investigated. The infrared light is tuned to the absorption maximum of water $(\lambda=2.94 \mu \mathrm{m}, 10 \mathrm{~ns})$, whereas the substrates used are transparent (glass, silicon). Thus a thin liquid water layer condensed on top of the contaminated substrate is rapidly heated. The pressure generated during the subsequent phase explosion generates a cleaning force which exceeds the adhesion of the particles. We examine the cleaning threshold in single shot experiments for particles sized from $1 \mu \mathrm{m}$ down to $300 \mathrm{~nm}$.
\end{abstract}

PACS 64.70.F- $\cdot 68.43 . V x \cdot 81.65 . \mathrm{Cf}$

\section{Introduction}

Laser Cleaning since its first appearance in the beginning of the 1990s is a topic of ongoing interest as the removal of smaller particles from very sensitive surfaces is of growing need. Different variants have been studied, which include among others Dry Laser Cleaning (DLC) [1, 2], Steam Laser Cleaning (SLC) [3-6], where additionally a thin liquid layer is deposited on the sample, and Matrix Laser Cleaning (MLC) [7], which involves a solid layer condensed onto the cooled substrate. While it is hard to prevent surface damage in DLC, SLC and MLC showed high cleaning efficiencies down to particle sizes as small as $50 \mathrm{~nm}$. Nevertheless a major drawback of these techniques is that they rely on absorption of laser energy in the substrate. This is not only a problem for glass surfaces in optics but also on inhomogeneous surfaces like metals on semiconductors, where huge

P. Frank $(\varangle) \cdot$ F. Lang · M. Mosbacher · J. Boneberg $\cdot$ P. Leiderer University of Konstanz, Fach M676, 78457 Konstanz, Germany e-mail: pascal.frank@uni-konstanz.de differences in absorption might appear and thus homogeneous cleaning is not feasible.

An approach to solve this problem is the use of an absorbing liquid or alternatively the tuning of the wavelength into a part of high absorptivity in the liquid. While the first proposal is hard to realize in the visible without the use of a dye from which new cleaning problems might appear, the second was realized for water at two different wavelengths. Allen et al. $[3,4]$ studied cleaning at $10.6 \mu \mathrm{m}$ where the absorption length in water is $20 \mu \mathrm{m}$. Zapka et al. [2,5] used an Er:YAG with the wavelength of $2.94 \mu \mathrm{m}$ where the absorption length is further reduced to $1 \mu \mathrm{m}$. While the examination of Allen et al. showed successful cleaning down to $1 \mu \mathrm{m}$, the experiments of Zapka et al. showed poor cleaning for $50 \mathrm{~nm}$ gold particles. Although in principle higher absorption should allow one to clean more efficiently, no further cleaning was studied to our knowledge. Thus we repeated the cleaning experiments but with focus on the standard particles used for cleaning measurements, namely polystyrene with sizes between $300 \mathrm{~nm}$ and $1 \mu \mathrm{m}$.

\section{Experimental setup}

The infrared steam laser cleaning setup (see Fig. 1) is composed of two major parts: a system to condense vapor onto a sample and an intense laser, which is focused on the sample.

The vapor deposition system allows us to deposit uniform liquid layers on a sample. We use glass (infrasil quartz glass and standard microscope slides) and doubleside polished silicon substrates, contaminated with colloidal particles (polystyrene; PS) with narrow size distributions (standard deviation below 10\%). Particle sizes from $1 \mu \mathrm{m}$ to $300 \mathrm{~nm}$ are used. They are applied onto the surface by spin 


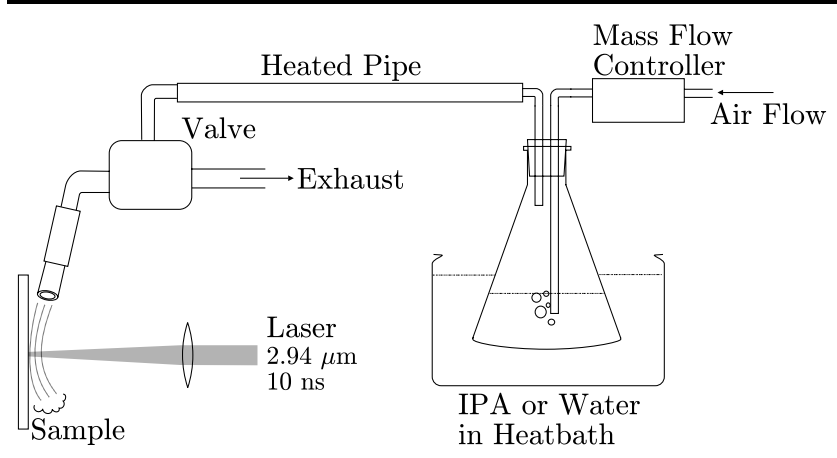

Fig. 1 Setup of the condensation system for steam laser cleaning experiments. The sample for infrared laser cleaning is accessible from both sides. The reflectometer and the in-situ microscope are not shown for the sake of clarity

coating. To obtain the infrared light, an Optical Parametrical Oscillator has been set up, which allows one to reach the wavelength of $2.94 \mu \mathrm{m}$. The used $\mathrm{LiNbO}_{3}$ crystal is pumped by an Nd:YAG-Laser ( $\lambda=1064 \mathrm{~nm}, \mathrm{FWHM}=12 \mathrm{~ns})$ and has an output power of about $20 \mathrm{~mJ} /$ pulse.

The liquid layer is formed by leading air through heated water (approx. $60^{\circ} \mathrm{C}$ ). The saturated vapor is then blown over the surface of the sample at room temperature, so that condensation sets in. Some efforts have been made to ensure a homogenous condensation process without the formation of droplets being splashed on the surface. So the pipe is constantly heated, and a valve ensures a constant gas flow through the liquid.

To control the condensation process, a reflectometer is set up [8]. The actual cleaning is monitored with an in-situ microscope with a 10 -fold magnification. The sample is accessible from the front as well as the back side so that cleaning experiments from both sides of the substrate are possible.

To elucidate the underlying physical process, we measured the velocity of the ablation plume. For that purpose, we installed a sheet of light (Ar-ion laser, $488 \mathrm{~nm}, 200 \mathrm{~mW}$ ) at a distance of $2.5 \mathrm{~mm}$ from the surface of the substrate. The light that is scattered by the ablation plume consisting of water vapor and droplets is then recorded by a photomultiplier.

\section{Results}

For the cleaning procedure, water is condensed onto the surface for about one second. This leads to an equivalent layer thickness around $1 \mu \mathrm{m}$. The subsequent infrared laser pulse heats the liquid rapidly. As the heating rate is sufficiently fast and the deposited energy is large, the liquid gets superheated, and the liquid-vapor phase transition is therefore explosive [9]. The generated pressure is high enough to detach and remove the particles. The process is schematically
Fig. 2 The infrared laser pulse is absorbed in the applied liquid. The liquid is heated above the critical temperature $\left(>300^{\circ} \mathrm{C}\right)$, which causes a phase explosion. The generated pressure results in cleaning forces, which exceed the adhesion. The sketch shows the process upon front side illumination. The experiments are also done by irradiation through the substrate from the back side
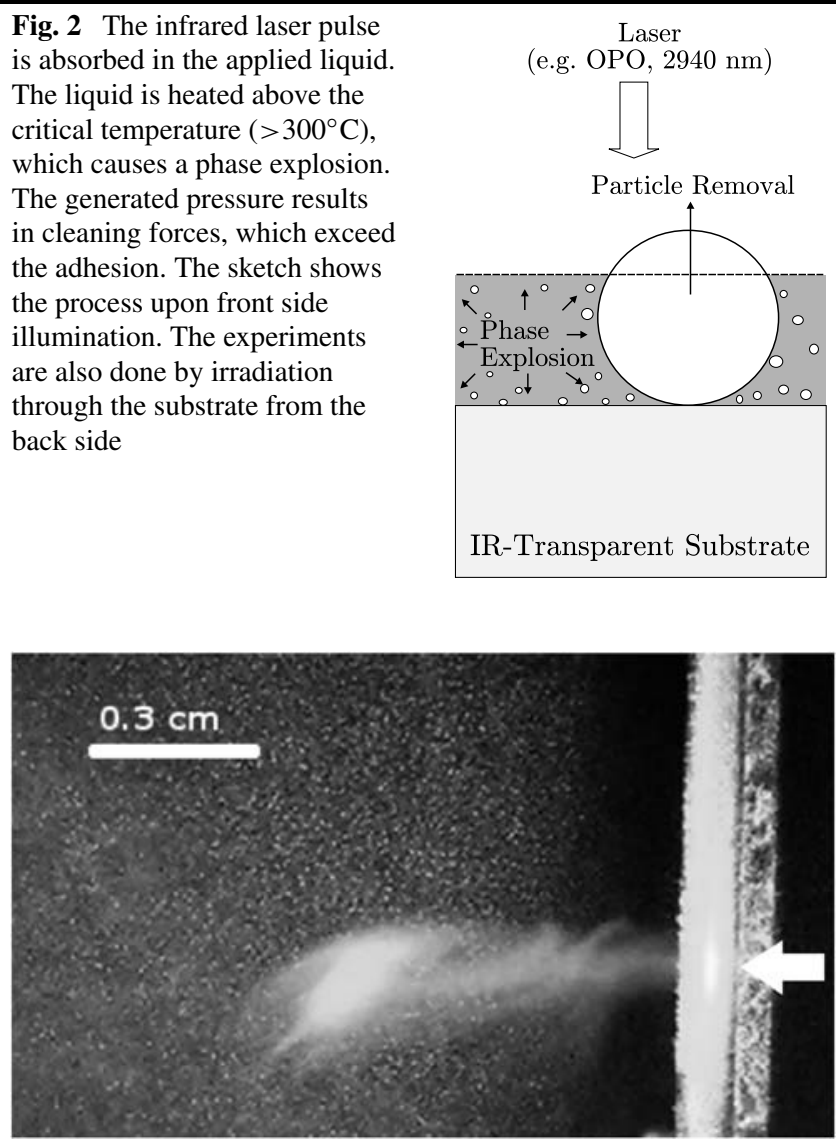

Fig. 3 Photograph of the ablation plume resulting of the phase explosion of a liquid water film on a substrate after irradiation with an infrared laser pulse $(2.94 \mu \mathrm{m}, 10 \mathrm{~ns})$. The focus of the beam is indicated on the slightly inclined cover glass. The laser is incident from the back side of the substrate, thus the right side in the picture. The background noise is due to the illumination

shown in Fig. 2, and a photograph of the phase explosion of a water film on a cover glass is depicted in Fig. 3.

The microscope image of a cleaned area of a glass substrate is shown in Fig. 4. The laser beam profile is slightly elliptical, the laser fluence at the center of the beam was $700 \mathrm{~mJ} / \mathrm{cm}^{2}$. The lateral extension of the cleaned area is about $1 \mathrm{~mm}$.

For the analysis of the threshold fluence necessary for cleaning, we focus on single shot experiments despite redeposition which might take place after one cycle cleaning.

From the extension of the spot, together with the known laser profile, it is possible to determine the threshold of the laser fluence. To identify the beam profile, the energy is measured, while a knife edge is moved through the beam at the focus. As shown in Fig. 5, the profile can well be assumed to be Gaussian. The maximal intensity is calibrated with a pinhole of $300 \mu \mathrm{m}$ diameter. Consequently the measured width of a cleaning spot refers to the threshold fluence.

The cleaning threshold of different sizes of PS particles on fused silica (Infrasil quartz glass, $1 \mathrm{~mm}$ thick; see Fig. 6) 


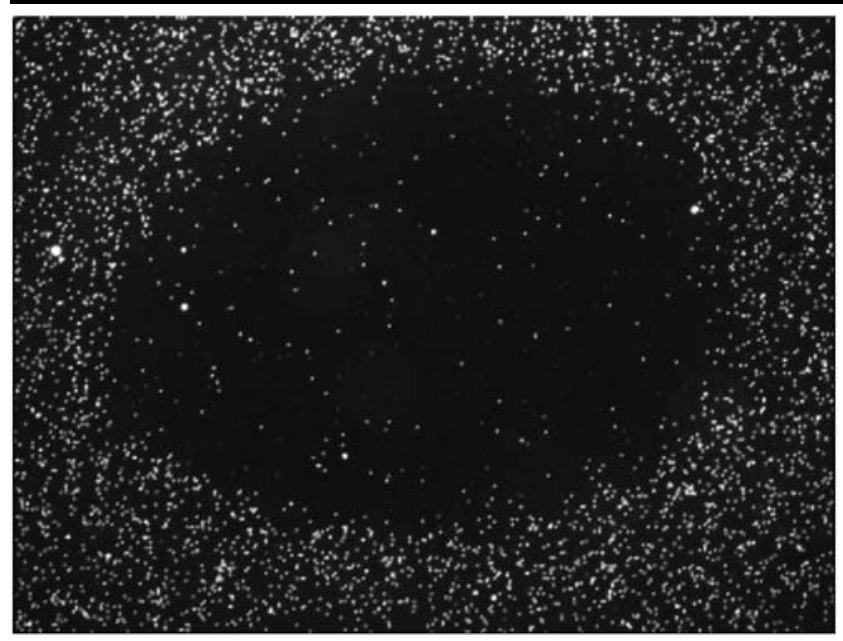

Fig. 4 Microscope image of the cleaned area of a glass slide contaminated by $510 \mathrm{~nm}$ polystyrene particulates. The lateral extension of the cleaned area is about $1 \mathrm{~mm}$

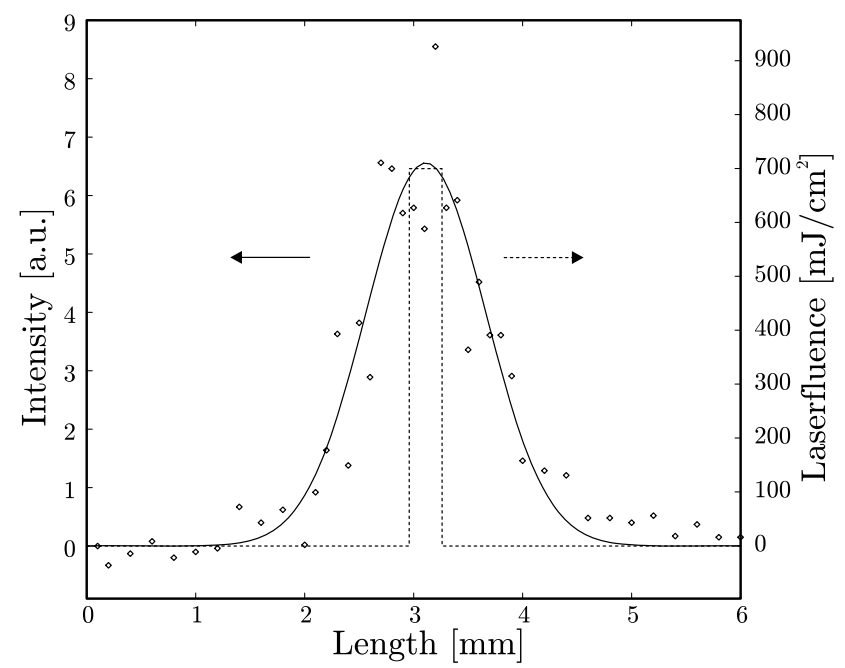

Fig. 5 Dots: Beam profile measured with a knife edge; Solid line: Gaussian fit of the profile, the maximum is calibrated with a pinhole $(0.3 \mathrm{~mm}$, dashed line). The width of a cleaning spot then refers to the threshold fluence which is necessary for cleaning

and microscope slides $(0.5 \mathrm{~mm}$ thick; see Fig. 7$)$ have been determined. The uncertainties depicted in the figures reflect the fact that a small error in the measurement of the spot diameter leads to a greater error in the laser fluence due to the steep Gaussian beam profile. The cleaning threshold for both substrates decreases with increasing particle size. The transmission of infrared light through the used substrates is sufficiently high to allow cleaning even with laser incidence from the back side. The cleaning threshold decreases upon irradiation of the back side of the transparent substrate. The values are corrected regarding the transmission through the substrate, reflection of the liquid layer is neglected.

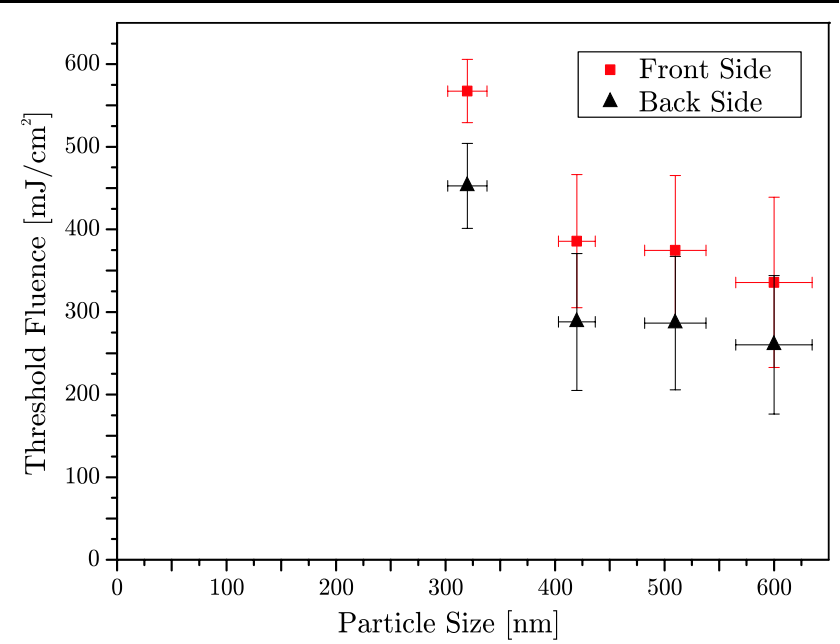

Fig. 6 Single shot cleaning of PS particles of a fused silica substrate. The threshold fluence is right above the fluence, which is necessary to cause a phase explosion of an $1 \mu \mathrm{m}$ thick water layer heated up to the critical temperature (approx. $200 \mathrm{~mJ} / \mathrm{cm}^{2}$ )

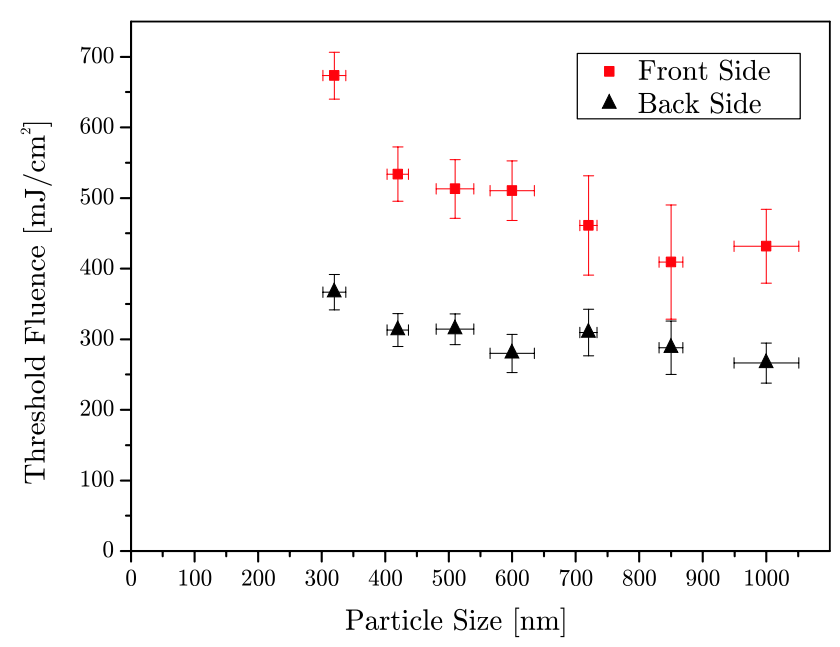

Fig. 7 Threshold fluence for PS particles for single shot cleaning of glass. As the energy is deposited underneath the particles, in the case of back side cleaning, the threshold decreases

The measurements regarding the velocity of the ablation plume are shown in Fig. 8. The scattered light increases after 15 to $55 \mu$ s after the laser pulse, depending on the laser energy fluence. From the time of flight the velocity of the plume can be derived. It increases from 50 to $150 \mathrm{~m} / \mathrm{s}$ with increasing laser pulse energy.

\section{Discussion}

Former SLC experiments have shown a universal cleaning threshold for the particle sizes tested [6, 8]. The cleaning forces obviously exceed the adhesion forces, so that 


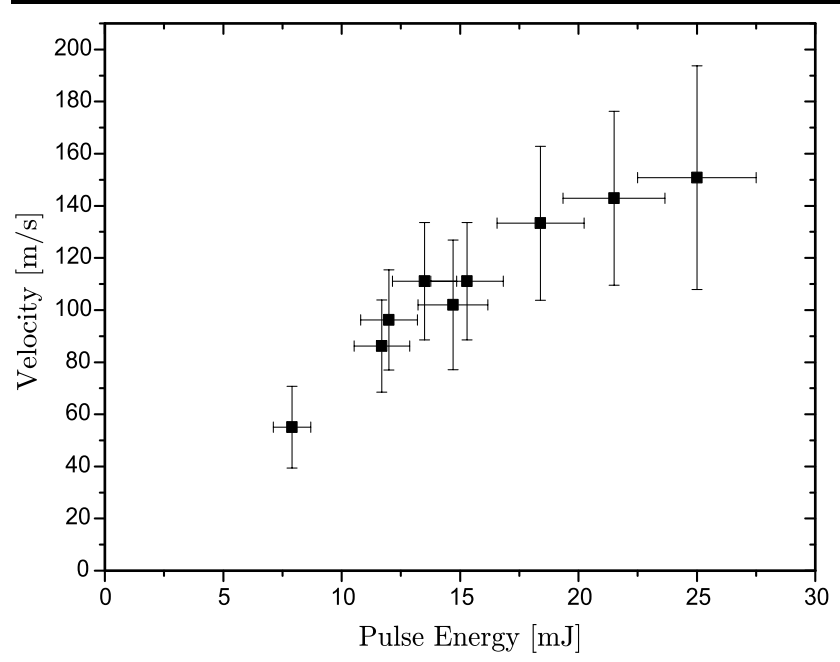

Fig. 8 Velocity of the ablation plume consisting of water droplets and vapor after the phase explosion on the cleaning substrate

if the energy is high enough to induce the phase transition at the interface of the heated substrate, cleaning takes place. This is obviously not the case in infrared cleaning. Here the cleaning threshold fluence increases for smaller particles. This is the expected behavior as despite the adhesion forces decrease for smaller particles following van-derWaals-theory (proportional to the radius $r$ ), the force acting on the particle to detach it scales with the area it can affect on (i.e., proportional to $r^{2}$ ). The detachment therefore gets harder for smaller particles [3]. The increase indicates also that the cleaning forces during the phase explosion are in the order of the adhesion forces of the particles and increase with increasing laser energy density. This is confirmed by the measurement of the plume velocity, which also increases with increasing pulse energy (see Fig. 8).
The comparison of the threshold for front side and back side cleaning indicates that the phase explosion takes place in a directional manner which eases particle removement from the back side. We assume therefore that the cleaning forces gets higher if the maximal heated liquid volume is underneath the particles.

Comparing our results to the experiments of Zapka et al. $[2,5]$ is quite difficult due to the fact that the contaminant particles (gold particles) used there are absorbing. Thus the temperature and the phase explosion will be inhomogeneous, which might influence the removal in a negative way.

In conclusion pulsed infrared laser cleaning allows the removal of particles at least down to $300 \mathrm{~nm}$ on nonabsorbing materials. In this way it might get feasible to clean inhomogeneous surfaces like chromium masks as well.

Acknowledgements Financial support by the Center of Applied Photonics (CAP) at the University of Konstanz and the Deutsche Forschungsgemeinschaft (DFG) project number LE 315/22 is gratefully acknowledged.

\section{References}

1. A.C. Engelsberg, Dissertation, Rensselaer Polytechnique Institute, 1988

2. W. Zapka, W. Ziemlich, A. Tam, Appl. Phys. Lett. 58, 20 (1991)

3. K. Imen, S. Lee, S. Allen, Appl. Phys. Lett. 58, 203-205 (1991)

4. S. Lee, K. Imen, S. Allen, Appl. Phys. Lett. 61, 2314-2316 (1992)

5. A. Tam, W. Leung, W. Zapka, W. Ziemlich, J. Appl. Phys. 71, 3515-3523 (1992)

6. M. Mosbacher, V. Dobler, J. Boneberg, P. Leiderer, Appl. Phys. A 70, 669-772 (2000)

7. J. Graf, B. Lukyanchuk, M. Mosbacher, M.H. Hong, C.T. Chong, J. Boneberg, P. Leiderer, Appl. Phys. A 88, 2 (2007)

8. F. Lang, M. Mosbacher, P. Leiderer, Appl. Phys. A 77, 117-123 (2003)

9. A. Vogel, V. Venugopalan, Chem. Rev. 103, 577-644 (2003) 